\title{
PERFORMANCE ANALYSIS OF THE AP AND THE SSC AND DESIGN OF THE SYSTEM OVERLOAD CONTROLS OF INDIA'S C-DOT DIGITAL SWITCHING SYSTEM
}

\author{
B. Madhavi and Anurag Kumar \\ Department of Electrical Communication Engineering \\ Indian Institute of Science \\ Bangalore, 560 012, INDIA \\ anurag@vigyan. ernet. in
}

\begin{abstract}
Government of India's Centre for Development of Telematics (CDoT) has developed a modular, distributed telephone switching system. There is an ongoing effort to model, analyse, and optimise the teletraffic performance of this switching system. In this paper we report the methodology and results of a part of this work. We use analytical queuing models to estimate the capacities of the Administrative Processor, and the Space Switch Controller. We formulate the system overload control problem as a constrained optimisation problem, solve the problem, and use simulations to evaluate various adaptive overload control algorithms.
\end{abstract}

\section{Introduction}

Government of India's Centre for Development of Telematics(C-DoT) has developed a modular, distributed, digital switching system that will eventually be capable of supporting upto 16,000 ports and an attempt rate of 300,000 calls/hour (see Figure 1). There has been an ongoing effort to analyse and optimise the performance of the various processor based modules of this switching

- system. A detailed simulation model was used to estimate the capacity of a Base Module to design an overload control for it, and to show how its capacity can be improved. This work was reported in [2]. In this paper we report on the methodology we have used to (i) analyse the performance of two piocessor based modules, namely the Administrative Processor (AP) and the Space Switch Controller (SSC), and (ii) design the system level overload controls.

The AP and the SSC perform call processing tasks and also participate in various lower priority activities such as testing and audits. We identify the various performance criteria that mnst be met for each of these classes of tasks in each of the two modules. We then develop a queueing model, with three priority levels, that applies to both the modules. The analysis of this queueing model reduces to that of a standard "vacation model". Numerica computations from well known Laplace transform formulae yield the desired performance measures. We provide numerical results based on preliminary rea time measurements obtained from C-DoT.

We then turn to the problem of designing the system level overload control, i.e., an overload control strategy that protects all the call processing modules. At variance with the ad hoc techniques that have previously been adopted for such problems, we formulate the problem as one of minimising average call set-up time while keeping throughput as large as possible. This yields a contrained nonlinear minimisation problem. The problem is easily solved and scheme is developed for on-line tuning of the controls (i.e., an adaptive overload control). Simulations are used to study the performance of the algorithm. Preliminary results show that the algorithm gives good transient and steady state performance.

This paper is organised as follows In Section $\mathbf{2}$ we give an overview of the C-DoT DSS architecture. In Sections 3 and $\mathbf{4}$ we describe the performance analysis of SSC and AP. In Section $\mathbf{5}$ we briefly present the methodology for designing the system level overload controls.

This paper is a brief summary of the methodology and results. The detail are available e'sewhere. See [2], [7], [6].

\section{Overview of C-Do'T DSS Architecture}

The C-DoT DSS has a modular and distributed architecture (Figure 1) consisting of a number of processor based modules, namely an Administrative Module (AM), a Central Module (CM), and a number of Base Modules (BM)

The AM provides the functions of overall system initialisation, systemwide maintenance, call routing and trank allocation. The system administration consoles are connected to the Input-Output Processor (IOP), which interfaces to the system ria the AM.

The CM consists of a Space Switch (SS), the Space Switch Controller (SSC), and a Central Message Switch (CMS). The space switch provides the digital paths for voice connections between BMs. The SSC allocates and deallocates

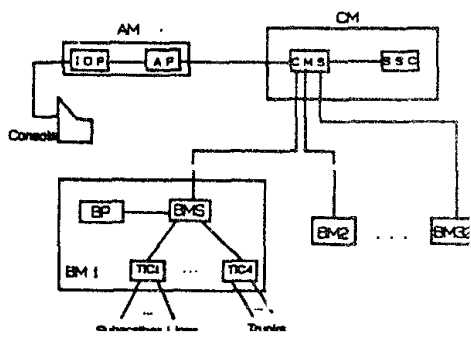

Figure 1: Architecture of the C-DoT DSS

inter-BM speech paths through the space switch. The CMS is basically a simple packet switch, and is connected to the BMs and the SSC by $64 \mathrm{kbps}$ links and to the AM by a $128 \mathrm{kbps}$ link. The various modules coxnmuaicate with each other by sending messages that are switched between the links by the CMS A simple stop-and-wait ARQ [8] protocol is used on each link. End-to-end message integrity is maintained by an end-to-end go-back-n ARQ protocol [8].

Lines and trunks terminate on the Terminal Interface Controllers (TICs) in the Base Module. Each Base Module comprises four TICs, each providing access to $\mathbf{1 2 8}$ subscriber lines or trunks. Hence the maximum configuration of $32 \mathrm{BM}$ supports 16,000 lines/trunks. A TTC performs fnnctions such as origination detection, digit reception, answer detection, digit outpuking etc. for the line and trunk terminations. A BM also contains a Tine Switch (TS) (time slot interchanger), a Time Switch Controller (TSC), a Biase Message Switch (BMS) and a Base Processor (BP). The time switch is part of the overall time-space-time switching fabric, and is also used for swiiching intraBM calls. The BMS provides message switching between the TICs and the $\mathrm{BP}$. The BP processes call stimuli in the BM

The processor modules run the CDOS operating system. This is a multitasking operating system, in which processes interact by passing messages to each other. The setup of a call involves the interaction of several software processes running in the processors of the various modules. Each of these processes can either be eternal or dynamic. Eternal processes are created during system initialization, whereas dynamic processes are created on demand by the operating system and they terminate on completion of their task (typically on the completion of a call). The processes communicate between themselves through messages. As mentioned above, when the processes are on different modules, the interprccessor messages are switched by the CMS.

Each process has associated with it a message queue. When a process gets its turn to run on the processor, it processes a message in its message queue. Upon completion of this work, the process joins the back of a queue of processes waiting for service at the processor. The operating system permits process priorities, but the priority is nonpreemptive. Further, the queue of messages waiting at a process may also he ordered by message priorities. Note here that, although prorities between software processes are nonpreemptive, hardware priority is preemptive. Thus, for example, an interrupt by a link controller will interrupt a softwase process. This has important implications for central resources (e.g., AP and SSC) that must communicate frequently with all the other modules.

The details of a typical call set up are given in [2]. 


\section{Performance Analysis of the Space Switch Controller}

\subsection{The SSC and its activities}

Recall from Section 2 that the Central Module houses the Space Switch which is a part of the overall time-space-time switching fabric and is used for switching inter-BM calls. The SSC is concerned with establishing and tearing down paths through the Space Switch. This is the only call setup related activity of the SSc.

The SSC communicates with the various module processors through mes sage transmissions and receptions that cause hardware interrupts to the SSC Each link interrupt needs service from an interrupt service routine that is exe"cuted by the SSC processor. The protocol used for message exchange between processors is such that, each message results in 4 interrupts to a processor. A message reception/transmission is followed by its link acknowledgement, then by an end-bend acknowledgement and finally by a link acknowledgement for the end-to-end acknowledgement. Recall that hardware interrupts are served at the highest priority and are preemptive.

In the current software version, some maintenance and audit processes are run in the SSC. The SSC periodicailly receives sanity monitor punches (SMPs) from the various processors, namely the BPs, the AP, the CMS. These are served at $a$ higher priority khan calls. In addition, the SSC performs tests that are served at alower prioriky than call processing tasks. There are three types of tests: (i) Sequential Mernory Access test, (ii) Voice Path test, and (iii) Link test. Lastly the SSC is required to perform auditing functions that are also lower in priority than call processing. One such audit function is the detection and release of hanging buffers.

\subsection{SSC processor occupancy as a function of load}

We have seen from the previous section that tests and audits are performed only when the processor is not involved in the processing of calls, sanity punches or interrupts. Such work is stid to be deferrable. There are three non deferrable tasks in the SSC, namely, processing of calls, processing of sanity punches and processing of interrupts. We shall refer to the fraction of time that the SSC is busy performing non-defertable tasks as SSC occupancy.

Let

$\rho=$ SSC processor occupancy (due to non deferrable work)

$\lambda=$ Busy Hour Call Attempt (BHCA) rate

$\sigma=$ Fraction of attempts at the BMs that arc good attempts (i.e., not partial dials, no-dials, etc.)

$\lambda_{C}=$ Arrival rate of requests for path setup and tear down $=2 \sigma \lambda$

$b_{\text {setup }}=$ Service time of a path set up restitest

$b_{\text {teardown }}=$ Service time of a path tear down

$\lambda_{Y}=$ Arrival rate of link interrupts to the SSC processor

$b_{\text {irat }}=$ Internugt service intue PSMP $=$ Occupancy of the $\$$ SSC processor due to sanity monitor punches

We note that $b_{\text {setup }}, b_{t a r i o w n}$ and $b_{\text {in: }}$ are modelled as fixed times and are obtained from measurements. It follows that the SSC proceror occupancy is given $b v$

$$
\rho=\rho_{S M P}+\sigma \lambda\left(b_{\text {aetup }}+b_{\text {ieardown }}\right)+\lambda_{I} b_{\text {int }}
$$

Note that the interrupt arrival rate $\lambda_{I}$ comprises the interrupts related to calls and also the interrupts due to other messages. Recall that the message protocol is such that each message (sent or received) causes 4 link interrupts. Each call generates 3 messages at the SSC: a switch path request from the AP, a termination message from the $\mathrm{SSC}$ to the destination $\mathrm{BP}$, and a switch path release message to the SSC.

i.etting

$\lambda_{Y_{0}}=$ interrupt rate due to non-call related messages

we have,

$$
\lambda_{I}=12 \sigma \lambda+X \mid
$$

$\lambda_{I_{0}}$ is easily calculated by accounting for the message traffic due to sanity monitor punches, and the various tests. It turns out that, for a $32 \mathrm{BM}$ configuration, and for the current software version, $\lambda_{I_{0}}=150$ per second. For details see [7].

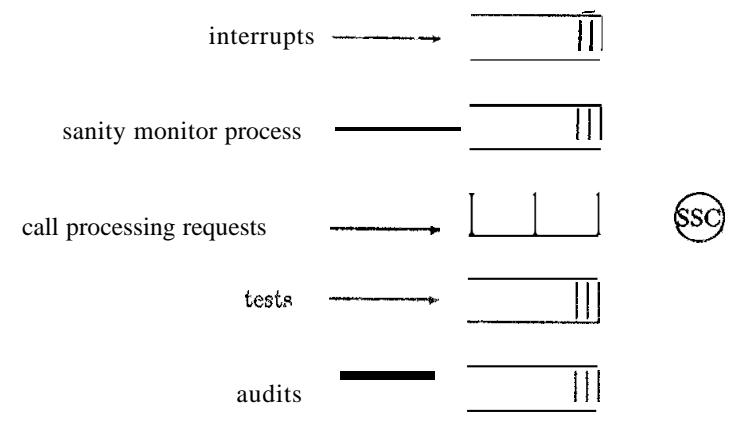

Figure 2: Five priority queuing model for the SSC

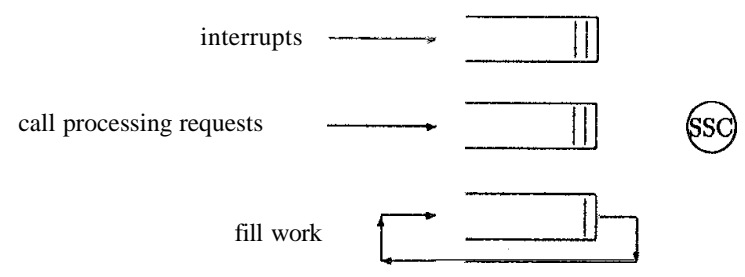

Figure 3: Three priority queuing model for the SSC

\subsection{Queuing model for the SSC}

From Section 3.1, it is clear that the SSC processor has to serve 5 types of customers at different priority levels. This would yield a model as shown in Figure 2. The SSC occupancy due to sanity monitor process is of the order of $1-2 \%$. We remove the SMP out of the model by inflating all service times by a factor of $1 / 0.98$ or $1 / 0.99$

Note that tests and audits are performed at the lowest priority, but are not indefinitely deferrable. There is an attempt to perform tests and audits in segments of $10 \mathrm{~ms}$. approximately. Though this traffic is not always present, we model it as though it is always queued for service at the $\mathrm{SC}$ and call it "fill work". It then becomes a point of interest to know the ict number of till work segments (of $10 \mathrm{~ms}$. each) that can be served by th $\mathrm{SC}$ in a given time.

Thus we have a 3-priority queue model consisting of int rrupts, path setups/tear downs and fill work segments as shown in Figure" .

Note that interrupts can occur either during $a$ call servic time or the service time of a fill work segment. These interrupts receive preesuptive service at the highest priority. This suggests that we can have a further simplified 2-priority
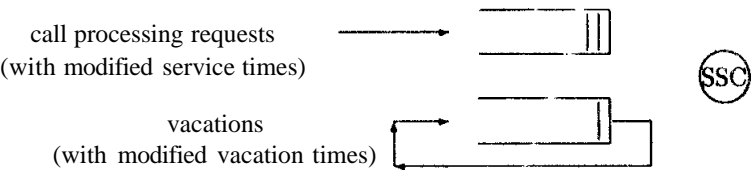

Figure 4: Two priority queuing model for the SSC

model consisting of calls and fill work segments both of whose service times are modified appropriately to take into account the effect of the preempting interrupts.

Moreover, since fill work segments are performed only in the absence of calls we can model these as nonpreemptible "vacations" taken by the server whenever the call queue is empty.

If $\lambda_{C}$, the arrival rate of path setups and teardowns into the SSC, is assumed to be Poisson, then the above modification of service times due to interrupts can be done exactly, and we have the standard $M / G / 1$ queue with vacations as the model for SSC [4]. This final model is shown in Figure 4. 


\section{3.: Pesormance criteria}

We first note that the call sojourn times at the SSC directly add up to the end-to-end set up delay. Hence an important performance measure is switch path request sojourn time at the SSC

Though tests are deferrable, there is a requirement that they cannot be delayed by more than a specified amount of time. It follows from our 2-priority yueue model that this requirement will be met if there is a limit on the length of call busy periods. Similarly, a certain number of audits must be completed within a specified amount of time. Thus completion time for a cycle of tests and audits is another performance measure of interest.

These performance measures generate the following performance require meuts, where $t_{1}, t_{2}$ and $t_{3}$ are specified time constraints, and $\alpha_{1}, \alpha_{2}, \mathbf{0 3}$ are probabilities ( close to 1 ) with which these constraints must be met.

$$
\begin{aligned}
& P(\text { Call setup or teardown time }\left.<t_{1}\right)>\alpha_{1} \\
& P\left(\text { Call busy period }<t_{2}\right)>\alpha_{2} \\
& P\left(\text { Cycle time for } \mathrm{F} \text { fill work segments }<t_{3}\right)>\alpha_{3}
\end{aligned}
$$

We see that the performance requirements are specified in terms of probability distributions of the corresponding random variables. Hence we resort to the determination of Laplace Stieltjes Transforms [4] for the distributions of

1. Sojourn times in the call processing queue

2. Busy periods in the call processing queue

3. Completion time for $F$ fill work segments

These are then numerically inverted using a numerical technique [3] to obtain the probability measures.

\subsection{Analysis of the queuing model}

3.4.1 Modification of call service times and fill work segments to incorporate interrupts

Let $\mathrm{X}$ denote the call service time random variable, $Y$ the interrupt service time random variable, and $V$ the vacation time random variable. Thus, for the workload model in Section 3.2,

$$
X= \begin{cases}b_{\text {setup }} & \text { with probability } 1 / 2 \\ b_{\text {teardown }} & \text { with probability } 1 / 2\end{cases}
$$

Further, $\boldsymbol{Y}=b_{\text {int }}$ with probability 1 . Letting

$b_{f i l l}=$ fill work segment length $(=10 \mathrm{msec}$ in the current system)

we have $\mathrm{V}=b_{f i l}$ with probability 1 .

Proceeding more generally, however, let $\tilde{x}(s)$ denote the Laplace Stieltjes Transform (L.S.T) of the cumulative distribution function (c.d.f.) $X(t)$ of $X$. Let $X_{I}$ denote the call service time modified bv hardware interrupts, and define $X_{I}(t)$ and $\tilde{x}_{I}(s)$ analogously. Similarly, define $Y(t), \tilde{y}(s)$ and $V(t), \tilde{v}(s), V_{I}, V_{I}(t)$, Assuming that the interrupts arrive in a Poisson process independent of the call arrival process, we now proceed to find $\tilde{x}_{I}(s)$

Since the interrupts receive preemptive service at the highest priority, the interrupt service queue is just an $\mathrm{M} / \mathrm{G} / 1$ queue. Let $G_{I}$ denote its busy period distribution, and $\tilde{g}_{I}(s)$ the L.S.T. of $G_{I}$ (4]. It follows that

$$
\tilde{g_{I}}(s)=\tilde{y}\left(s+\lambda_{I}-\lambda_{I} \tilde{g_{I}}(s)\right)
$$

Conditioning on the length of the unmodified call service time $X$, we get,

$$
\begin{aligned}
\tilde{x}_{I}(s) & =\int_{0}^{\infty} e^{-s x} \sum_{k=0}^{\infty} \frac{\left(\lambda_{I} x\right)^{k}}{k !} e^{-\lambda_{I} x}\left(\tilde{g}_{I}(s)\right)^{k} d X(x) \\
& =\int_{0}^{\infty} e^{-x\left(s+\lambda_{I}\left(1-\tilde{g}_{I}(s)\right)\right)} d X(x)
\end{aligned}
$$

1.e.,

$$
\tilde{x}_{I}(s)=\tilde{x}\left(s+\lambda_{I}\left(1-\tilde{g}_{I}(s)\right)\right)
$$

Similarly, we have

$$
\tilde{v}_{I}(s)=\tilde{v}\left(s+\lambda_{I}\left(1-\tilde{g}_{I}(s)\right)\right)
$$

For the work load model discussed above,

$$
\begin{aligned}
& \tilde{x}(s)=0.5 e^{-s b_{\text {cetsp }}}+0.5 e^{-s b_{\text {tea }}}=e^{-s b_{\text {int }}} \\
& \tilde{y}(s)=e^{-s b_{\text {sit }}}
\end{aligned}
$$

Also it follows that,

$$
\begin{aligned}
& E X_{I}=E X-E X \frac{\lambda_{I} b_{\text {int }}}{1-\lambda_{1} b_{i n t}}=\frac{\left(b_{\text {setup }}+b_{\text {ieardown }}\right) / 2}{1-\lambda_{I} b_{\text {int }}} \\
& E V_{I}=E V+E V \frac{\lambda_{I} b_{i n t}}{1-\dot{\lambda}_{I} \dot{b}_{\text {int }}}=\frac{b_{i} i L}{1-\dot{\lambda}_{I} \dot{b}_{\text {int }}}
\end{aligned}
$$

\subsubsection{Call sojourn times}

Now we have an $M / G / 1$ vacation model for the $\mathrm{SSC}$ with arrival rate $\lambda_{c}$, service time L.S.T. $\tilde{x}_{I}(s)$ and vacation time L.S.T. $\tilde{v}_{X}(s)$. Let $T_{C}$ be the random variable for the sojourn time of a call at the SSC. Let $\tilde{t}_{C}(s)$ denote the L.S.T. of the c.d.f.. $T_{C}(t)$ of $T_{C}$. Let $T_{C_{0}}$ and $\tilde{t}_{C_{0}}(s)$ denote the random variable and its L.S.T. for call sojourn time in an $M / G / 1$ queue without vacations. It follows from the Pollaczek - Khinchine formula [4] for sojourn time in an $M / G / 1$ queue (without vacations), that

$$
\tilde{t}_{C_{0}}(s)=\frac{s\left(1-\rho_{C I}\right) \tilde{x}_{I}(s)}{s+\lambda_{C}-\lambda_{C} \tilde{x}_{I}(s)}
$$

where,

$$
\rho_{C I}=\lambda_{C} E\left(X_{I}\right)
$$

Clearly $\rho_{\mathrm{C} I}$ is the processor occupancy due to calls and the interrupts that occur during call processing.

It now follows from the stochastic decomposition property of the $M / G / 1$ queue with server vacations $[1]$ that

$$
\begin{aligned}
\tilde{t}_{C}(s) & =\tilde{t}_{C I}(s) \frac{1-\tilde{v}_{I}(s)}{s E V_{I}} \\
& =\frac{s\left(1-\rho_{C I}\right) \tilde{x}_{I}(s)}{s+\lambda_{C}-\lambda_{C} \tilde{x}_{I}(s)} \frac{1-\tilde{v}_{I}(s)}{s E V_{I}}
\end{aligned}
$$

where $\frac{1-\tilde{v}_{r}(s)}{v_{Y}}$ is the L.S.T. of the c.d.f. of the residual lifetime random variable of $\frac{s E v}{V_{I}[9] \text {. }}$

\subsubsection{Call busy periods}

For calculation of call busy period distribution, we consider a vacation in which at least one arrival has occurred.

Note that the presence of vacations in the model causes the elongation of call busy periods because an arrival to an empty system does not immediately start service, but waits for the completion of the vacation time Other arrivals may occur during this residual vacation and these will contribute to the following busy period. Let $G_{C v}$ dencte the random variable for the busy period due to calls arriving during such a vacation and $\tilde{g}_{C V}(s)$ be its L.S.T.

Let $\tilde{g}_{C}(s)$ denote the L.S.T. of the call busy period distribution in the model without vacations. We have [4],

$$
\tilde{g}_{C}(s)=\tilde{x}_{I}\left(s+\lambda_{C}-\lambda_{C} \tilde{g}_{C}(s)\right)
$$

Conditioning on $V_{I}$ and then unconditioning we get

$$
\tilde{g}_{C I}(s)=\frac{\tilde{v}_{I}\left(\lambda_{C}-\lambda_{C} \tilde{g}_{C}(s)\right)-\tilde{v}_{I}\left(\lambda_{C}\right)}{1-\tilde{v}_{I}\left(\lambda_{C}\right)}
$$

where $\tilde{v}_{I}(s)$ is as in the previous section.

3.4.4 Cycle time for $F$ fillwork segments (vacations)

Let $U$ denote the random variable denoting the time until one cycle of $F$ fllwork segments is completed and a fresh cycle starts and let $\tilde{u}(s)$ denote its L.S.T.

A vacation is followed by a call busy period if arrivals take place during the vacation. Otherwise, the server goes on another vacation. Owing to the assumption of Poisson arrivals, we have a renewal process with a renewal epoch defined at the start of a vacation.

Let $Z$ denote the random variable for the interrenewai time and $\tilde{z}(s)$ its L.S.T. Conditioning on the vacation length random variable $V_{I}$, and recalling (7) $\tilde{g}_{C}(s)$ from Equation 13, we have

$$
\tilde{z}(s)=\tilde{v}_{I}\left(s+\lambda_{C}\left(1-\tilde{g}_{C}(s)\right)\right)
$$

Finally, $\tilde{u}(s)$ is obtained from $\tilde{u}(s)=\tilde{z}(s)^{F}$ 


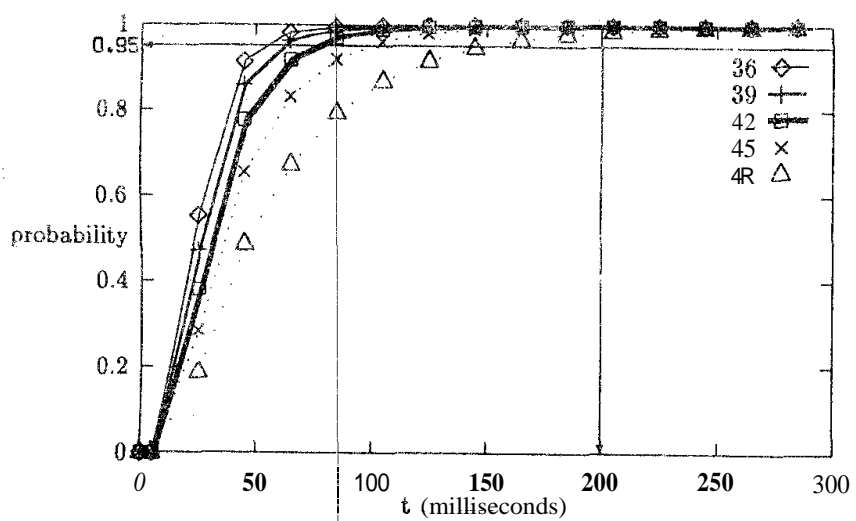

Figure 5: SSC performance: Probability (path setup/teardown delay $<t$ ) vs. t. $\alpha_{1}=0.95, t_{1}=200 \mathrm{~ms}$.

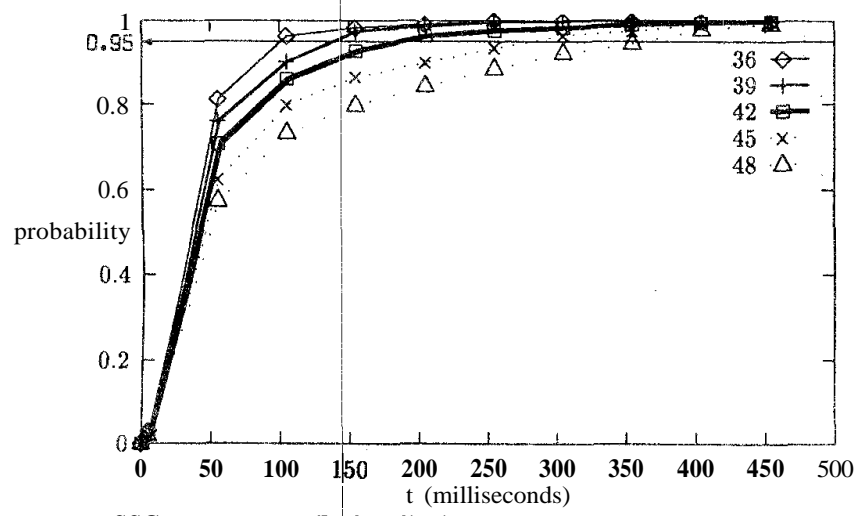

Figure 6: SSC performance: Probability (call processing busy period $<t$ ) vs. $t$ $\alpha_{2}=0.95, t_{2}=5 \mathrm{rec}$.

\subsection{Occupancy measurements in the $\mathrm{SSC}$}

We note here briefly our resolution of an anomaly that was obnerved by C-DoT engineers when they measured SSC processor occupancy. Whereas processor 'occupancy should increase linearly with $\lambda$, as is clear from Equation 1, the measurements yielded a nonlinear, convex, increasing curve.

Upon investigation, it turned out that the measurement process was meast.ring the fraction of time the SSC queue in nonenipty. This is what we have denoted as $\rho_{C I}$ (Equation 11), id is clearly nonliriear in $\lambda$. The reason for the nonlinearity is clear: as $\lambda$ increases, so does $\lambda_{I}$, thus causing an increase on the effective mean service time of a call request (i.e., $E X_{I}$ ).

\subsection{Numerical Results}

The transforms obtained in the previous section were inverted using a numerical inversion technique for Laplace transforms [3]. The numerical data used are as follows: $\sigma=0.735, b_{\text {setup }}=10 \mathrm{~ms}, b_{\text {teardown }}=6 \mathrm{~ms}, b_{i n t}=0.6 \mathrm{~ms}, b_{f i l l}=$ $10 \mathrm{~ms}, t_{1}=200 \mathrm{~ms}, t_{2}=5$ seconds, $t_{3}=10$ seconds, $F=100, \alpha_{1}=\alpha_{2}=$ $\alpha_{3}=0.95$.

The cumulative distribution functions for the various performance measures were obtained for a set of $\lambda$ values corresponding to $70 \%, 75 \%, 80 \%, 85 \%$ and $90 \%$ occupancy of the SSC. These values of $\rho$ correspond, respectively, to $\lambda=36,39,42,45,48$ call attempts per second.

Figure 5 shows the probabillty distribution of the call setrip/teardown delay in the SSC for the various values of BHCA mentioned above. It is clear that space switch path request delay requirements are easily met upto an attempt rate of $48 \mathrm{calls} / \mathrm{sec}$, i.e., $172,800 \mathrm{BHCA}$, which corresponds to $90 \%$ processor arceupan, $y$.

Figure 6 shows the distribution of call processing busy period. Clearly this performance requirement does/not constrain SSC capacity at all.

Figure 7 shows the cycle time for completing 100 fill work segments. This graph shows that at $\mathbf{4 5} \mathrm{calls} / \mathrm{sec}$ this requirement is met, but at $\mathbf{4 8}$ calls/sec it a not.

Owing to certain operational requirements, the possibility of adding other kinds of work to the SSC, and due to just plain caution, it is not considered appropriate to operate at above $70 \%$ occupancy. All performance requirements are, of course, met at this occupancy corresponding to a load of $129,600 \mathrm{BHCA}$

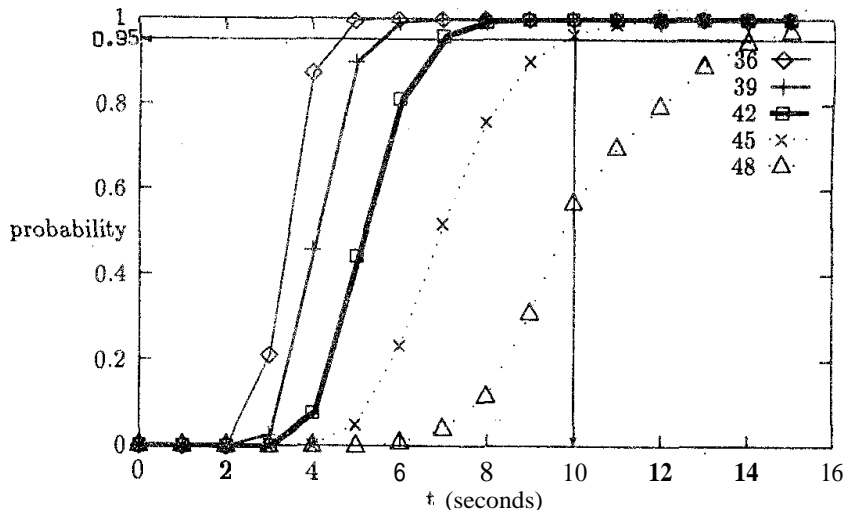

Figure 7: SSC performance Probability (completion time for 100 fill-work segments $<t$ ) vs $t \quad \alpha_{3}=095, t_{3}=10$ sec

\section{Performance Analysis of the Administrative Processor}

From a modelling perspective, the AP is very similar to the SSC.

\subsection{The AP and its activities}

Recall from Section 2 that the AP is connected to the CMS through a 128 kbps link and is the interface between the switch and the system administration consoles. Call processing work at the AP involves routing and allocation/deallocation of trunk resources. In addition, the AP acts as a conduit for flow of billing and traffic information from the BMs to the secondary storage at the Input Output Processor(IOP). The AP communicates with the other module processors through messages that are switched by the CMSs. The AP involves itself in processing the hardware link interrupts generated by these message transfers. Like every other processor, the AP participates in basic maintenance activities which include the reception of sanity puzches from its copy (the AP is duplexed), the SSC, the IOPs and the Alarm and Display Unit (ADP). The AP in addition performs andits during idle time.

\subsection{AP occupancy}

We define the activities of call processing, sanity punch processing and interrupt processing its constituting the nondeferrable work at the AP. Deferrable work comprises the collection and delivery or billing/traffic records to the $10 \mathrm{~F}$. and also audits.

AP occupancy is the fraction of time the AP spends on nondeferrable work The methodology for expressing the occupancy as a function of the call attempt rate is similiar to that of the $\mathrm{SSC}$.

Since the discussion in this section is entirely about the AP, we can adopt the same notation as in Section 3, without causing any confusion

Let

$b_{\text {alloc }}=$ service time for routing and trunk allocation

$b_{\text {dealloc }}=$ service time for trunk deallocation

Each intermodule call generates 3 messages at the AP: a route-request from the originating $\mathrm{BM}$ to the $\mathrm{AP}$, a path setup request from the AP to the SSC, and finally, on call completion, a resource deallocation request from the originating BM to the AP. It follows, as in the case of the SSC, that

$$
\rho=\rho_{S M P}+\sigma \lambda\left(b_{a l l o c}+b_{\text {sealloe }}\right)+\lambda_{I} b_{\text {int }}
$$

and

$$
\lambda_{1}=120 \lambda+\lambda_{I_{d x m p}}+\lambda_{I_{0}}
$$

where $\hat{\lambda}_{\lambda_{\alpha m m}}$ is the interrupt rate due to billing and subscriber monitoring dumps from the BMs to the IOP via the AP, and has the form

$$
\lambda_{I_{d u m p}}=0.3 \sigma \lambda+0.16 \lambda
$$

The coefficients in this equation are obtained from analysis of the AP in. terrupts due to dumps, and $\lambda_{I_{0}}=85$ per second for a 32 BM configuration[7]. 


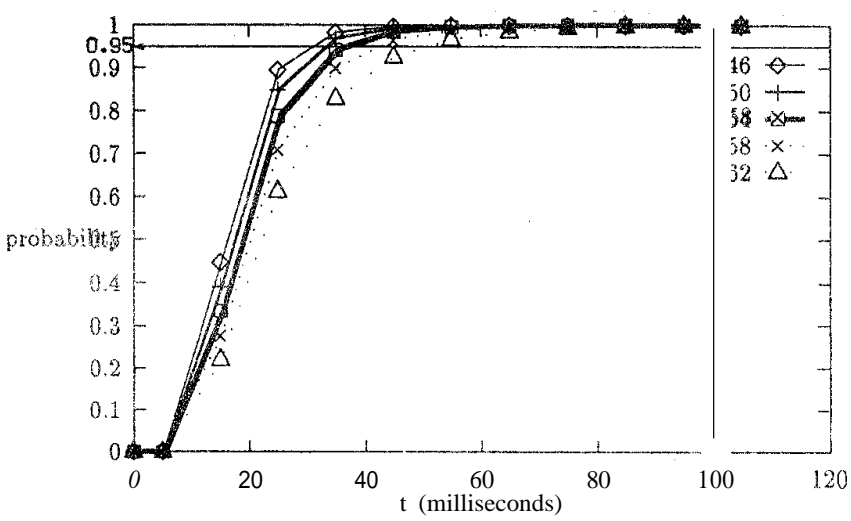

Figure AP performance: Probability (call sojourn time at A? $<t$ ) vs. $t \cdot \alpha_{1}=$ $0.95 . t_{1}=100 \mathrm{~ms}$

\subsection{Queuing model for the AP}

For the purposes of modelling, work at the AP is considered to be at three priority levels:-

1. Interrupts, caused by message transmission and reception over the inks, are served at hardware priority level. These are preermbive in nature.

2. Call processing work that involves routing arid trunt allocation/deallocation is served at the highest software priority level

3. Billing, traffic dumps and audits are served at the lowest priority.

Thus we again have a three priority mode: as with the SSC. Again, we treat the low priority work as fill work that is done in $10 \mathrm{~ms}$. segments. The analysis is identical to that in Section 4.

The performance measures identified are call soloum times and the probability that $F$ fill work segments are completed within a given duration, which translate into the following performance requirements:-

$$
\begin{array}{r}
P\left(\text { call sojourn time at the } A P<t_{1}\right)>\alpha_{1} \\
P\left(\text { cycle lime for F fill work segments }<t_{2}\right)>\alpha_{2}
\end{array}
$$

\subsection{Numerical results}

The numerical data used for obtaining occupancy arid performance measures are as follows: $\mathscr{\sigma}=0.735, b_{\text {alloc }}=7 \mathrm{~ms}, b_{\text {dealloc }}=3 \mathrm{~ms}, b_{\text {int }}=0.6 \mathrm{~ms}, b_{\text {fill }}=$ $10 \mathrm{~ms}, t_{1}=100 \mathrm{~ms}, t_{2}=3600 \mathrm{sec}, a:=\alpha_{2}=0.95$

In Figure 8 the c.d.f. of call sojourn time in the AP is plotted for $\lambda=46$, $50,54,58,62$ calls $/ \mathrm{sec}$, which correspond, respectively, to $\rho=0.60,0.65,0.70$, $8.75,0.80$. It is seen from the graph that sojourn time requirements are met êven upto $\rho=0.80$

In Figure 9 we pint the probability of performing $100 \mathrm{~N}$ fill work segments in 3600 seconds $r$ isus $N$, for $A=46,50,54,58,62 \mathrm{calls} / \mathrm{sec}$. The amount of fill work to be performed in one hour depends on $A$, since the billing and traffic dumps are traffic. relat: Conseouently, the value of $F$ in the performance requirement (Equation $1 B$ ) depercis on $A$. For each $\lambda$ the corresponding value of $F$ is show as an abscissa in Figue 9 , the values increasing with BHCA. It is clear that only for $\lambda=46(p=0.6)$ is the performance requirement met.

It follows that the capacity of the $\mathrm{AP}$ is $165,600 \mathrm{BHA}$ at $60 \%$ processor occupancy.

\section{System Overload Control}

In this section, we propose and partialiy develop a methodology for designing an overload control strategy for the entire switch. We only have space here to provide a summary of our work; for details see [7] and [6].

Recall that the capacity of each processor module corresponds to a maximum occupancy. If the load offered causes an occupancy exceeding this, an overload control mechanism must respond quickly and attempt to shed the excess load. The objective of such a load shedding strategy will be to maximise carried load, while giving satisfactory performance to all the carried load.

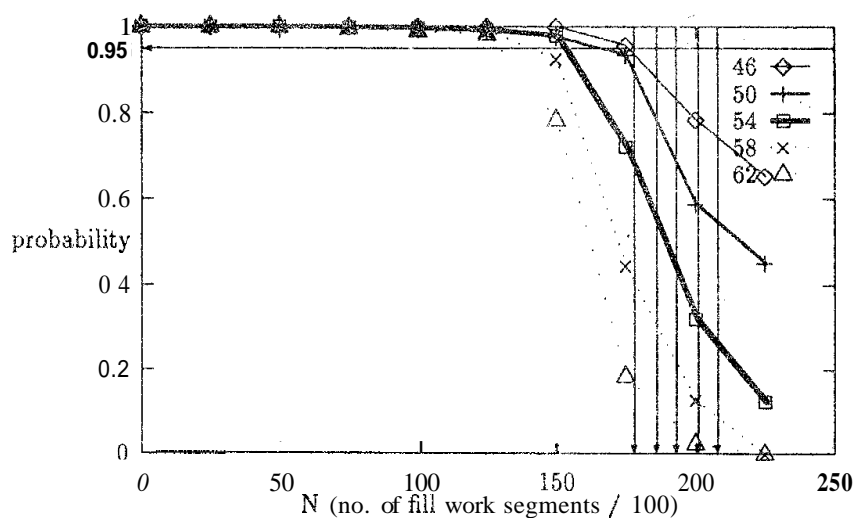

Figure 9: AP performance: Probability (number of till work segments done in $60 \mathrm{~min}>100 \mathrm{~N}$ vs. $N . \alpha_{2}=0.95$. (The vertical arrows point to the fill work requirement at each BHCA. This requirement increases with BHCA.)

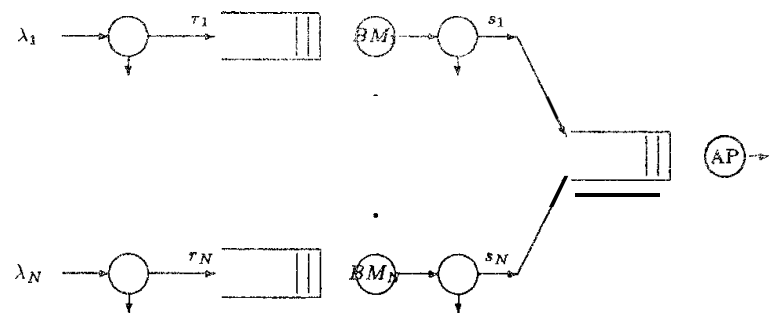

Figure 10 Queuing model for the overload control problem. The "wive"-like symbols denote load shedding points

\subsection{Formulation and solution of the overload control prob- lem}

In the work that we report here, we have taken the first step towards devising an integrated control strategy for protecting all the processor based modules. For simplicity, we begin by considering only the BPs arid the AP. This motivates the model in Figure 10 with which all the subseruent discussions are concerned.

There are $N$ BMs. In Figure $10, \lambda_{i}$ denotes the rate of calls entering $B M_{i}$ Prior to each BM, there is a pre - dial control that controls the rate into $B M$ to $r_{i}(<A$,$) . Between each B M$ and the $A P$ there is possibly a post - dial control that further limits the rate $r_{i}$ from $B M_{i}$ to $s_{i}\left(\leq r_{i}\right)$. Thus the total input rate into the $A P$ is $\rangle_{i=1} s_{i}$

The mean service times by $b$ and $a$. We are giver $0<\rho_{B P}^{*}<1$ and $0<\rho_{A P}^{*}<1$, such that the occupancies of each. $B P$ and $A P$ must be maintained less than $\rho_{B P}^{*}$ and $\rho_{A P}^{*}$ respectively. Thus, all the offered load can be handled by rach of the processors, if $\lambda_{i} \leq \rho_{B P}^{*} / b$, for all $i$, and $\sum_{i=1}^{N} \lambda_{i} \leq \rho_{A P}^{*} / a$. It follows that the maximum carried load $\ddot{\gamma}$ for given $\left(\lambda_{1}, \ldots, \lambda_{N}\right)$ is given by $\vec{\gamma}\left(\lambda_{1}, \ldots, \lambda_{N}\right)=$ $\min \left(\left(\sum_{i=1}^{N} \min \left(\lambda_{i}, \rho_{B P}^{*} / b\right)\right), \rho_{A P}^{*} / a\right)$.

The problem is to decide at which points control should be exercised and for a given set of values $\left(\lambda_{1}, \ldots, \lambda_{N}\right)$ what the values of $\left(r_{l}, \ldots r_{N}\right)$ and $\left(s_{1}, \ldots, s_{N}\right)$ should be. We will impose the constraint that the throughput of the system should be as large as possible, i.e., $\bar{\gamma}\left(\lambda_{1}, \ldots, \lambda_{N}\right)$. Clearly there are many ways of shedding load to achieve this maximum throughput.

In a post-dial scheme, such as that of AT\&'s's 5 ESS, though the contrcl cart react rapidly to an overload, it is clear that under sustained overload, serving calls at She BMs oniy to reject them later is wasteful of the BM capacity. It would be good to have a mechanism that rejects calls right at their entry point to the BM, i.e., a pre-dial control. In such 2 case, we can have reduced dialtone delays. In fact, our simulation results have shown that even the transient performance of tho post dial control is not superior to the controls we devise. 
At variance with the adhoc techniques used in earlier and existing switches, we have given a novel theatment for over. sad control by formulating it, as a problem of finding the smount of load to be accepted at each BM. so that average end-to-end call selup delay is minimised, while keeping: the throughput as large as possible $\left(1 . e\right.$, at $\left.\bar{\gamma}\left(\lambda_{1}, \ldots, \lambda_{N}\right)\right)$. We shall assume that all the call rejection is done before the BPs.

Let $N, 6$, and a be as kdefined earlier in this section. Let

$x_{i}=$ call arival rate to $B P_{i}$

$r_{i}=$ accepted call ratet an $B l_{i}$

We will hirst assume that $x_{i}$ is a load that can be safely handled by $B M_{i}$ (i.e, $x_{i}=\min \left(\lambda_{i}, p_{B ;}^{*} / b\right)$, Assume the presence of a pre-dial control for all the BMs that lets in loaris that are within their capacities. Thus essentially we will first show how to further limit the load to protect the AP.

Now it can be shown $([7],[6])$ that if the mean delay curves of the BPs are convex, and nondecreasing, then in order to minimise mean call setup delay while keeping throughput as large as possible (i.e, $\min \left(\sum_{i=1}^{N} x_{i}, \rho_{A F}^{*} / a\right)$ ), the optimal predist control has the following form

$$
r_{i}^{*}=\min \left(x_{i}, r^{*}\right), \quad 1 \leq i \leq N
$$

where $r^{*}$ is the solution of

$$
\sum_{i=1}^{N} \min \left(x_{i}, r^{*}\right)=\min \left(\sum_{i=1}^{N} x_{i}, p_{A P}^{*} / a\right)
$$

Observe that the form of the optimal solution naturally lends itself to a threshold control implem'ntation. The ideal throughput of such a controller is given $b y$

$$
\begin{cases}r_{i}=x_{i} & \text { if } x_{i}<r^{*} \\ r_{i}=r^{*} & \text { if } x_{i} \geq r^{*}\end{cases}
$$

A threshold controller is realised as follows [5], and does not require knowledge of the $x_{i}$. Consider the presence of a token pool at the BM. Each call that enters the BM is accepted for service at the BM, only if the pool is not empty. The call takes away a token. Arriving calls that find the pool empty, are rejected. When the threshold is $r^{*}$, a token is added to the pool every $1 / r^{*}$ units of time. The token pool can hold a maximum of $M$ tokese. Thi ideal throughput is achieved with $M=\infty$. Thus to teep the thoughan is close to ideal as possible, a large stack is desirable. This, however, iseves open the possibility of too large a number of calls heing dispolche co the 130 in :

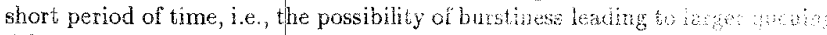
delays at the $\mathrm{BP}$ queue. Thus the value of the maximum town com in compromise between throughput and delay.

\subsection{On-line determination of the optima contret level}

We reed a control algorithm that obtains $r$ from on-line newsirements. The value of $r$ should closely track the optimal control $r^{*}$ as the offered had varies

For fixed $\left(\lambda_{1}, \ldots, \lambda_{N}\right)$, i.e., fixed $\left(x_{1}, \ldots, x_{N}\right)$, define $f(r)=\sum_{i=1}^{N} \min \left(x_{i}, r\right.$, Observe that $f(x)$ is the rate of calls reaching the AP when the threshold control at each of the BMs is set to $r$. The problem of obtaining $r^{*}$ (Equation 19) can now be formulated as that of obtaining the projection of the root of $f(r)-\rho_{A P}^{*} /\left(a=0\right.$ into the interval $\left[\rho_{A P}^{*} /(a N), \rho_{B P}^{*} / b\right][7]$. Note that "noisy" measurements of $f(r)$ can be obtained by measuring the occupancy of the AP over successive intervals. The control level is adjusted at. the ends of these measurement periods.

Thus the problem becomes one of finding a projection of the zero of a certain function $f(r)$ whose noisy values can be obtained for each value of its argument r. The same problem was obtained in the context of post-dial control of the CP in the 5ESS switch, and well known stochastic approzimation techniques have been appiied to it. by the first author In [5]. If $y_{k}$ is the measurement of $f\left(r_{k}\right)$. (i.e., at the $k^{t h}$ step of the iteration), the algorithm yields $r_{k+1}$ as hlows

$$
r_{k+1}^{-1}=\theta_{k+1}=\left[\theta_{k}+\sum_{i=k-W+1}^{k} r_{i}^{2}\left(y_{k+1}-1\right)\right]_{b / \rho_{B P}^{*}}^{(a N) / \rho_{A}^{*}}
$$

where [\}$_{i}^{\bar{E}}$ is the projection of the quantity in [.] into the interva! $[\theta, \bar{\theta}]$. The sequence $\left\{\hat{\theta}_{\boldsymbol{k}}\right\}$ is an interrhediate quantity whose interpretation can be found in [5].

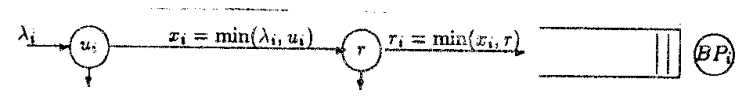

Figure 11 Schematic of independent BP and AP controls

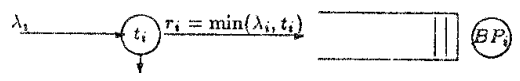

Figure 12 Schernatic of integrased Mi. and AP controls

\subsection{Integrated overload control for the BPs and the AP}

We have thus far assumed that the ofiered loads to each of the BMs are withi their capacities. Now suppose that the BP overload controls are also imple. mented as throhold controls (as shomn in Figure 11). Let

$u_{i}=$ the threshold $B_{i} P_{i}$ for protecting $B P_{i}$

$r=$ the hrosold thet is applied at each BP to protect the AP.

$\lambda_{2}-\ldots$ rate of calls offered to BP $P_{i}$.

Leting $t_{i}=\min \left(u_{i}, r\right)$, we see that both the controls can be integrated intc a single threshold control, as shown in Figure 12. This suggests that both the $A P$ and the $3 M$ in question work on the control algorithm independently and determine the values $r$ and $u_{i}$. The BM then applies a rate $\min \left(u_{i}, r\right)$ as the control.

\subsection{Siralation experiments and results}

A series of simulation experiments were done to study the proposed overloat control.

Each of the $N$ BMs is modelled as a single server quene. The BNB aro all identical, with equn service speeds. Calls arrive at the BM in 2 Poisson proces. Wo rodel a call as follows: Each call generates a random aumber of stmiti. A stimulus requires an exponentially distrbute nervice time of $b_{\text {sim }}$ milisecs. Wach stmulus, after its service time of $b_{\text {stim }}$ millises has ben

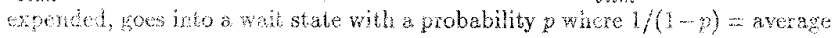

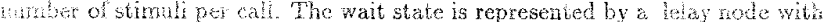
a dclay distribution that is exponertial with mean $\frac{1}{2}$ ms. Thus the mean service time of a call at the BMi is $b=\frac{b_{\text {atizx }}}{1-0}$.

The Administrative Processor $(A P)$ is moleled as a single server $\cdot / M / 1$ queue. Calls depraring from the queues of the various BMs enter this queue. Each call requires a millisecs at. the AP. We assume that each RM is offered a load all of which requires service at the AP aiso. Note that this assumption is not strictly true, since a BM is subjected to load due to both originations and terminations. Only originations eventually require service at the AP. Terminating calls at a BM require service oniy at the BM. Further work is needed to incorporate termination traffic into our model.

The numerical data used are as follows: $\mathrm{A}^{\prime}=30, \rho_{A P}^{*}=0.7, \rho_{B P}^{*}=$ 0.7 , Maximum value for the threshold counter $M=30, a=10 \mathrm{m \varepsilon}$, b.tior $=$ $20 \mathrm{~ms}, \boldsymbol{t}=500 \mathrm{~ms}, \boldsymbol{p}=0.9$, Measurement and control update interval $=5$ seconds.

We report here the results of an experiment that was done to study the effect of the location of the control points, (pre-dial or post-dial or both) and to siudy their relative performance with respect to the steady state and transient bchaviour. The loads on tire BMs were changed after every 3 minutes to study the adaptiveress of the control strutegy shown in Equation 20.

For the first 3 minutes, the Ioadson the BMs were $\lambda_{1} ., \lambda_{20}=3.5, \lambda_{21},, \lambda_{30}=$ 1. The correct level of control is 3.0 For the next 3 minutes, the loads were $\lambda_{1} \ldots \lambda_{30}=3.0$. The correct level of control is 2.33. For the last 3 minutes of the simulation, the loads were $\lambda_{2} . . \lambda_{30}=2.0$. The corret level of control is 4.5. 


\begin{tabular}{||c|c|c||}
\hline $\begin{array}{c}\text { control } \\
\text { scheme }\end{array}$ & $\begin{array}{c}\text { mean dial-tone } \\
\text { delay (ms.) }\end{array}$ & $\begin{array}{c}\text { mean delay } \\
\text { in AP (ms.) }\end{array}$ \\
\hline \hline I & 45.6 & 29.0 \\
II & 40.4 & 22.7 \\
III & 39.8 & 21.6 \\
\hline
\end{tabular}

Table 1: Mean dial-tone delay and mean delay in AP for the three overload control schemes.

Three alternatives were studied:

- Pre-dial rate control for the BM and a post dial rate control for the AP: This simulation was done to evaluate the performance of the post-dial scheme used in the 5ESS. Let this scheme be called Scheme I.

- Pre-dial rate control for the BM and a pre dial plus a post dial rate controlfor the AP: This control strategy was tested to see the advantage in having both a post-dial mechanism that may have the advantage of a better transient behaviour and a pre-dial mechanism that has better steady state behaviour. Under sustained oveload, the post-dial control will not have any effect since the pre-dial control would already have limited the loads to satisfy the AP requirements. We shall call this Scheme II.

- Pre-dial rate control for the BM as well as the AP: This is Scheme III.

We obtained plots of the control level and measured occupancy versus time for the $\mathbf{3}$ minute duration of each of the 3 experiments. Owing to lack of space we cannot show these here (see [6]). All three schemes track the correct control level, but there are differences in the convergence times. Scheme I being the slowest. We also obtained the mean dial-tone delay (this is taken to be the mean time for serving the first stimulus in the call model described above), and the mean delay in the AP for each experiment. These are shown in Table 1.

It is seen from Table 1 that Scheme I gives the worst performance in terms of average delays at the BP and the AP. Scheme 11 gives a performance close to that of Scheme III, but is slightly inferior. We now compare the schemes I and III. The strategy used in the 5ESS namely a pre-dial control and an independent post-dial control for the AP gives BP delays higher than that of the scheme that uses only a single pre-dial control for the BPs as well as the AP. This is of course expected since in Scheme I the BPs are unnecessarily handling load that is later rejected by the post-dial control. Scheme I, however, also gives a slightly higher delay at the AP. This is quite unexpected because a post dial scheme should react to a transient condition faster. The reason for the poor performance can be attributed to the finite convergence time of the algorithm in responding to the change in load. If we could have a hypothetical situation in which the AP could immediately detect an overload, then Scheme I would indeed give lower delays at the AP.

Work remains to be done to use this methodology have to develop a comprehensive overload control strategy, that protects all the processor modules, and accounts for all the types of traffic that modules are subjected to. In particular, we have not acconnted for termination traffic in the BPs; further our model does not incorporate the SSC. Our methodology establishes a conceptual framework, and it should not be difficult to incorporate these other factors.

\section{Acknowledgement}

We are grateful to several C.Do $\mathrm{T}$ engineers for answering patiently our many questions about the hardware and software architecture of the switch, and for providing us with various measurements. In particular, we wish to thank Mr. Venkatrajendran, Divisional Manager, Software, and the members of the Performance Engineering Group: Mr. Srinivasachar, Ms. Naseein Meriam, and Mr. Venugopal.

\section{References}

[1] S. W. Fuhrmann and R.B. Cooper, "Stochastic decomposition in an $M / G / 1$ queue with generalised server vacations", Operations Research, Vol.33, 1385 .

[2] B. Hareesh Kumar, "Performance analysis and optimisation of the C-DoT digital switching system", Master of Engineering Project Report, Dept. of Electrical Communication Engg., Indian Institute of Science, Bangalore, January 1990.

[3] D.L. Jagerman, "An inversion technique for the Laplace Transform," Bell System Technical Journal, Vol. 61, No. 8, pp 1995-2002, Oct. 1982.

[4] L. Kleinrock, Queueing Systems, Vol. I and II, John Wiley.

[5] Anurag Kumar, "Adaptive overload control of the central processor in a distributedgystem with a star topology," IEEE Transactions on Computers, October 1989.

[6] Anurag Kumar, B. Hareesh Kumar, and B. Madhavi, "Modelling and performance analysis of a digital switching system - case study of the C-DoT DSS", to appear in Journal of the Institution of Electronics and Telecommunications Engineers, 1991-92.

[7] B. Madhavi, "Performance analysis of the SSC and the AP and design of the system level overload controls in the C-DoT Digital Switching System," Master of Engineering project report, Dept of Electrical Communication Engg., Indian Institute of Science, Bangalore, January 1991.

[8] A. Tanenbaum, Compufer Networks, II edition, Prentice Hall, 1988

[9] R.W. Wolff, Stochastic Modelling and the Theory of Queues, Prentice Hall, 1989. 